\title{
Full Matching in an Observational Study of Coaching for the SAT
}

Ben B. HANSEN

\begin{abstract}
Among matching techniques for observational studies, full matching is in principle the best, in the sense that its alignment of comparable treated and control subjects is as good as that of any alternate method, and potentially much better. This article evaluates the practical performance of full matching for the first time, modifying it in order to minimize variance as well as bias and then using it to compare coached and uncoached takers of the SAT. In this new version, with restrictions on the ratio of treated subjects to controls within matched sets, full matching makes use of many more observations than does pair matching, but achieves far closer matches than does matching with $k \geq 2$ controls. Prior to matching, the coached and uncoached groups are separated on the propensity score by 1.1 SDs. Full matching reduces this separation to $1 \%$ or $2 \%$ of an SD. In older literature comparing matching and regression, Cochran expressed doubts that any method of adjustment could substantially reduce observed bias of this magnitude.

To accommodate missing data, regression-based analyses by ETS researchers rejected a subset of the available sample that differed significantly from the subsample they analyzed. Full matching on the propensity score handles the same problem simply and without rejecting observations. In addition, it eases the detection and handling of nonconstancy of treatment effects, which the regression-based analyses had obscured, and it makes fuller use of covariate information. It estimates a somewhat larger effect of coaching on the math score than did ETS's methods.
\end{abstract}

KEY WORDS: Graph algorithm; Matching with multiple controls; Network flow; Optimal matching; Quasiexperiment; Propensity score.

\section{INTRODUCTION}

During the 1995-1996 academic year, investigators from the College Board surveyed a random sample of high school junior and senior SAT takers to probe how they had prepared for the SAT, asking whether they had completed extracurricular test preparation courses, among other questions. Some $12 \%$ of respondents said that they had; the comparison of these to the remaining $88 \%$ comprised the observational study reported by Powers and Rock (1999).

Powers and Rock estimated coaching effects in several ways, most of which started from regression models and one of which involved matching. Matching was not their favorite approach: "By its nature," they lamented, matching "significantly reduces sample sizes," noting that their matched-pairs analysis matched only a fraction of the uncoached students to coached counterparts (1999, p. 99). Their disappointment seemed to extend from pair matching to matching in general, although it is not clear that it should have. As compared to $1: k$ matching or to matching with a variable number of controls (Ming and Rosenbaum 2000), pair matching is the least flexible and the least able to make use of a large reservoir of potential controls.

This article revisits Powers and Rock's matching problem using the most flexible approach applicable to it, namely optimal full matching (Rosenbaum 1991). Section 2 explains full matching and contrasts it with pair matching and similar designs. Full matching remedies the sample reduction problem, using all of the available sample, as none of Powers and Rock's preferred adjustments was able to do; simultaneously, it produces closer matches than do their methods. It turns out that full matching is in a sense too flexible (Sec. 2.4); Section 3 addresses this by modifying the technique to incorporate certain restrictions. Full matching, either with or without restrictions,

Ben B. Hansen is Assistant Professor, Statistics Department, University of Michigan, Ann Arbor, MI 48109-1092 (E-mail: ben.b.hansen@umich.edu; URL: www.stat.lsa.umich.edu/fbbh). The author thanks Paul R. Rosenbaum, as well as Luke Bulman, Tanya Henneman, Elizabeth A. Stuart, Yu Xie, and two anonymous referees for very helpful suggestions. He wishes to acknowledge the College Board, Donald E. Powers, and Donald A. Rock for graciously sharing the data from their SAT coaching study, Paul W. Holland for his kind encouragement and for his help in obtaining the data, and the National Science Foundation for material support (Postdoctoral Fellowship DMS-01-02056). does a better job with missing data, facilitates fully adjusted but simple comparisons of treated and control groups, and lays bare heterogeneities of treatment effect that regression analyses obscured.

The context of Powers and Rock's study was as follows. The Princeton Review has long claimed its students' average benefit to be 140 points in combined SAT score (Princeton Review 2004), and during the 1990s Kaplan Educational Centers claimed average benefits of 120 points (Zehr 2001). The coaching companies' figures appear to be based on studies conducted for them by outside firms (Princeton Review 2004); but because neither the studies nor methodological descriptions of them are published or publicly available, the integrity of their conclusions is difficult to assess. In contrast, Powers and Rock found much weaker coaching effects: about 20 points on the math section and 10 on the verbal. Their analyses assumed, among other things, constancy of coaching effects. Granting this and other premises, Powers and Rock's findings sharply refute those of the coaching companies. Section 4 will offer strong evidence against uniformity of coaching effects, however. Our full matching-based estimation of coaching effects, also presented in Section 4, relaxes this and others of Powers and Rock's assumptions, yielding new evidence on the College Board's and the coaching companies' competing claims. Section 5 abstracts from the coaching study to discuss matching for observational studies in general.

\subsection{Test Scores and Test Preparation in a National Sample}

The data to be analyzed derive from a stratified random sample of registrants for 1995-1996 administrations of the SAT-I test, details of which are given by Powers and Rock (1999). About 6,700 high school juniors and seniors received surveys asking whether and how they had prepared for the test; the replies of some 4,200 respondents were linked to the College Board's records of their scores on the 1995 or 1996 exams, as

(C) 2004 American Statistical Association Journal of the American Statistical Association

September 2004, Vol. 99, No. 467, Applications and Case Studies DOI 10.1198/016214504000000647 
Table 1. Selected Pretreatment Variables

\begin{tabular}{lccc}
\hline \hline Variable & $\begin{array}{c}\text { Range of } \\
\text { values }\end{array}$ & $\begin{array}{c}\text { Standardized } \\
\text { bias }\end{array}$ & $\begin{array}{c}\text { Percentage } \\
\text { of sample }\end{array}$ \\
\hline Math section & $20-43$ & -.1 & 18 \\
of PSAT & $45-51$ & .1 & 17 \\
& $52-57$ & -.1 & 16 \\
Mean SAT at & $58-80$ & .1 & 15 \\
respondent's & Not taken & .1 & 34 \\
first-choice & 787-987 & -.3 & 16 \\
college & $1,061-1,060$ & -.2 & 16 \\
& $1,124-1,336$ & .1 & 16 \\
Father's & No response & .3 & 16 \\
education & High school & .0 & 36 \\
& A.A. or B.A. & -.4 & 40 \\
Average & Graduate & -.1 & 26 \\
math grade & No response & .4 & 25 \\
& "Excellent" & .1 & 9 \\
Foreign & "Good"-"fail" & -.1 & 35 \\
language & No response & .1 & 59 \\
years taken & 0-2 & -.3 & 6 \\
\hline
\end{tabular}

well as scores on previous SAT-I or PSAT tests and their answers to the Student Descriptive Questionnaire (SDQ), which all SAT-I registrants are asked to complete. By their responses to questions about extracurricular SAT preparation, respondents split into a treated and a control group, and the data describe the results of a classical quasiexperiment (Campbell and Stanley 1966).

Nineteen in twenty of the survey respondents actually took the spring 1996 or fall 1995 exam for which they had registered. The analysis given below restricts itself to these 3,994 students, using the corresponding SAT scores as outcome measures. Thus the record gives coaching status and SAT outcomes for all students in the sample to be analyzed; among the additional measures, each available for some fraction of the students, are pretest scores, racial and socioeconomic indicators, various data about their academic preparation, and responses to a survey item that, by eliciting students' first choices in colleges, recovered an unusually discriminating measure of students' educational aspirations. In all, there are 27 pretreatment variables.

The coached and uncoached groups differ appreciably in these recorded measures - as do high and low scorers on the SAT. Table 1 offers some illustration of this, giving overall incidences of various covariate attributes and comparing their relative incidences in the coached and uncoached groups. (The statistic here used to effect these comparisons is the standardized bias, given for a variable $v$ by $\left(\bar{v}_{t}-\bar{v}_{c}\right) / s_{p}$, where $\bar{v}_{t}$ and $\bar{v}_{c}$ are the average values of $v$ in the treatment and control groups, respectively, and $s_{p}^{2}$ is the pooled within-group variance in $v$.) Yet the table shows only five covariates; the analysis must address biases on all 27 of them.

\subsection{Missing and Misleading Data in Regression and in Subclassification}

In regression-based adjustment, the simplest way to handle missing data on a covariate is to reject cases without complete information. In adjustment based on matching or stratification, the method of first resort is to merge "missing" with an appropriate level of the covariate, or to treat it as a category unto itself. Thus missingness becomes part of the profiles according to which study subjects are sorted into strata or matched sets. Good stratifications, then, will tend to group subjects that are comparable in terms both of observed covariate values and of covariate missingness.

Powers and Rock's (1999) study follows the norms of regression analysis rather than of stratification, rejecting all cases with missing covariate values. Of the seven statistical analyses they report having done, one used about an eighth of the available sample, three more used about half, another two used three quarters, and only one, the so-called "Belson model," used more than $90 \%$ of it. The Belson model was an outlier in another respect: Its estimate of the effect of coaching on math scores was closer to 30 points than the 15 or 20 found in the other analyses. And the difference of the treated and control groups' mean SAT scores is greater for the whole of the sample $(41 \pm 5$ for SAT-M, $9 \pm 5$ for SAT-V; $n=3,994$ ) than for the half of the sample used by three of Powers and Rock's analyses $(35 \pm 7$ for SAT-M, $6 \pm 7$ for SAT-V; $n=1,876$ ). The partly missing observations are decidedly unlike a randomly selected subset of the sample; to the contrary, their removal from an the analysis is likely to bias the result.

To illustrate how a stratification-based analysis might begin to address this problem, consider simple stratifications along the one or two covariates that most threaten to confound the comparison of treated subjects to controls. With the College Board coaching data, race and socioeconomic status (SES) variables best fit this description. The one race variable sorts subjects into eight ethnic categories, with only $6 \%$ of observations missing. Several of these groups are quite small, and collapsing seems in order. Given the education setting of the study, it is natural (1) to sort observations into an AsianAmerican category (9\%), an underrepresented minority category (8\% Black, 3\% Mexican American, $1 \%$ Native American, $1 \%$ Puerto Rican, $3 \%$ other Hispanic, $3 \%$ other), and White $(66 \%)$; and (2) to place the small fraction of item nonrespondents with the largest category, namely White. To account for SES, SDQ responses give three potential stratifiers to choose from among, namely parents' income and education levels of mothers and fathers. All three variables are probably measured with some error, but it seems that high school students are more likely to know and less likely to misreport their parents' education than their parents' income; and splitting the data into thirds at the $33 \%, 67 \%$, and $100 \%$ quantiles of mother's and of father's education levels, father's education better separates both PSAT-math and PSAT-verbal scores. We stratify the College Board coaching data by race and father's education level, grouping students into three categories of father's education, plus an additional category for students not reporting it. Call this the Race-by-SES (Race $\times$ SES) subclassification; Table 2 shows sizes and compositions of its subclasses.

The Race $\times$ SES subclassification adjusts for too few of the available covariates to be taken seriously as an adjustment unto itself, but it should be noted that it makes a promising beginning. For instance, the association between PSAT math scores (grouped as in Table 1) and coaching status is significant at the .05 level in the unstratified sample, but not in 
Table 2. Race $\times$ SES Subclasses: Sizes and Control-to-Treated-Subject Ratios

\begin{tabular}{lcc}
\hline \hline $\begin{array}{l}\text { Father's education } \\
\text { (by race category) }\end{array}$ & $\begin{array}{c}\text { Percentage } \\
\text { of sample }\end{array}$ & $\begin{array}{c}\text { Number of controls } \\
\text { per treated subject }\end{array}$ \\
\hline White, or no race reported & 26 & 21 \\
$\quad$ High school or less & 20 & 10 \\
A.A. or B.A. & 20 & 4.5 \\
Postcollege & 7 & 4.5 \\
$\quad$ Not reported & 72 & 8.2 \\
White (all) & & \\
Underrepresented minority & 11 & 11 \\
High school or less & 3 & 6.6 \\
A.A. or B.A. & 3 & 3.6 \\
Postcollege & 1 & 4.4 \\
Not reported & 19 & 7.2 \\
Underrepresented & & \\
minority (all) & & \\
Asian American & 4 & 3.8 \\
High school or less & 3 & 3.4 \\
A.A. or B.A. & 3 & 1.5 \\
Postcollege & .4 & 2.9 \\
$\quad$ Not reported & 9 & 7.0 \\
Asian American (all) & 100 & \\
All & & \\
\hline
\end{tabular}

the stratified sample, when evaluated with the Mantel (1963) score statistic; and after but not before subclassification along race and SES, a Cochran-Mantel-Haenszel test (Agresti 1990, sec. 7.4.6) fails to find significant differences between coached and uncoached students in terms of number of semesters taken of high school English and natural science, English and natural science grades, and grades in social science and math courses. Because other variables, such as overall high school grade point average (GPA) and reported parents' income, do not become balanced after stratification on SES and race, the analyst must make one or more additional adjustments taking the remaining covariates explicitly into account. To effect such an adjustment, Sections 3 and 4 of this article refine rather than replace the Race $\times$ SES subclassification, thus inheriting its gains.

Subclassifying in this way, we have rejected no observations. Placing subjects with partially missing data into subclasses dedicated to their missingness pattern, as we have done, can solve the missingness problem only if the unavailable covariate data are missing at random given those data that are not missing; but for the analogous strategy in regression contexts, namely casewise deletion, it is necessary that the observations with partial missingness be like a simple random subsample of the sample as a whole-which in the present case appears not to be true.

The strategy of creating missingness levels of covariates can also be used to construct propensity scores. It leads to propensity scores which, when matched or stratified upon, balance both covariate-missingness and observed-covariate profiles between treated and control groups (Rosenbaum and Rubin 1984, app.); it is well-suited to missingness patterns in which observations tend to lack only few of a large number of covariates. Such is the case here: On the 23 covariates other than pretest scores, only one third of the College Board sample have complete data, but two thirds are missing no more than two covariates, and nine tenths lack data on no more than six covariates. Our propensity score accommodates missing data in this way, in so doing retaining all 3,994 observations.
Adjustment by stratification encourages the analyst to focus on the data rather than a model for it, and this can be indirectly beneficial. With these data, for example, there is a temptation to regard as a pretreatment variable any PSAT or SAT score from a test sitting earlier than that of the posttest, in order to maximize sample size in a regression using pretest scores as covariates; recent regression-based studies of coaching for the SAT share in such a simplifying assumption (Briggs 2001; Powers and Rock 1999). But as it turns out, in the College Board's sample there are quite a few coached students who got their coaching even before taking their earliest SAT or PSAT: of 332 coached students reporting the years and months in which their test preparation courses began, one fourth started their courses before taking either the SAT or the PSAT. Treating as pretests prior test scores that did not genuinely precede the treatment, as Briggs' and Powers and Rock's studies do, may deny credit to coaching programs for gains that they produced. (For a more general discussion of this point, see Rosenbaum 1984.) The analysis to follow treats the 126 coached students with prior tests that did not precede their coaching, or could not be determined to have preceded it, the same as students without pretests: In refashioning the covariates for inclusion in a propensity score model, they are placed in a "pretest-missing" category. To enhance comparability of the groups, a similar accommodation is made with uncoached students: Those who have prior tests that only preceded their posttests by a period of less than 6 months are placed into a "pretest-missing" category, rather than a category based on groupings of pretest scores.

\section{CONVENTIONAL MATCHING AND ALTERNATIVES}

\subsection{Nearest Available versus Optimal Matching}

Most commonly, matchings join each treated subject to one or to a fixed number $k \geq 2$ of controls, and usually this matching is done by a so-called nearest available algorithm. Section 2.2 explores ramifications of matching treated and control subjects in only one, preset ratio. As a prelude to that discussion, this section reviews the distinction between optimal and nearest available matching.

Table 3 presents an artificial dataset modeled on an unpublished gender equity study conducted by the author. Men and women university scientists within various departments were to be compared in terms of their lab space assignments, but first it was necessary to match them on factors that might confound the comparison. The actual study matched on total grant funding and several other factors, but to simplify the illustration we consider grant funding alone.

Nearest available, or greedy, matching algorithms move down the list of treated subjects from top to bottom, at each

Table 3. A Gender Equity Matching Problem: Women and Men Scientists Are to Be Matched on Grant Funding

\begin{tabular}{lcccc}
\hline \hline & Women & & \multicolumn{2}{c}{ Men } \\
\cline { 1 - 2 } \cline { 5 - 5 } Subject & $\log _{10}$ (grant funding) & & Subject & $\log _{10}$ (grant funding) \\
\hline A & 5.7 & & V & 5.5 \\
B & 4.0 & & X & 5.3 \\
C & 3.4 & & Y & 4.9 \\
D & 3.1 & & Z & 3.9 \\
& & &
\end{tabular}


step matching a treated subject to the nearest available control, which is then removed from the list of controls available at the next step. Matchings are made at a given stage without attention to how they affect possibilities for later matchings. In the equity matching problem posed in Table 3, a nearest available algorithm for pair matching would first match $\mathrm{A}$ to $\mathrm{V}$, then $\mathrm{B}$ to $\mathrm{Z}$, $\mathrm{C}$ to $\mathrm{X}$, and finally D to Y, for a total "cost" (sum of absolute differences in log Grant Funding) of 3.6. Having matched A to V, $\mathrm{Z}$ is the nearest available potential match for $\mathrm{B}$, but matching $\mathrm{B}$ to $\mathrm{Z}$ is in fact "greedy," in that it forces $\mathrm{C}$ and/or $\mathrm{D}$ to be more poorly matched at the next stage. In contrast, optimal matching algorithms optimize global, rather than local, objectives. The optimal solution for the problem of pairing each of Table 3's women with one of its men joins $\mathrm{A}$ to $\mathrm{V}, \mathrm{B}$ to $\mathrm{X}, \mathrm{C}$ to $\mathrm{Y}$, and D to Z, for a total cost of 3.4.

For pair matching with a large reservoir of controls, greedy algorithms often do nearly as well as optimal algorithms (Rosenbaum and Rubin 1985). But absent an excess of available controls, or with unfortunate orderings of the list of treated subjects, greedy algorithms can do much worse than optimal ones.

\subsection{The Weakness of Fixed-Ratio Matching: Using More Controls Leads to Larger Biases}

Returning to the coaching study, let us match coached and uncoached students first as pairs, and then in fixed proportions $1: k$, letting $k$ grow until all controls have been matched, and let us compare these alternative matchings to one another. Because optimal matches are never worse, and often better, than greedy matches, we generate each match using optimal methods.

Surely the best $1: 1$ match is less likely than any $1: k$ match, $k \geq 2$, to join a treated subject to a control that differs appreciably from it; and surely it follows that among all $1: k$ matches, $k \geq 1$, an optimal 1:1 match most reduces the bias of treatment to control group comparisons. Yet it would be rash to prefer $1: 1$ matches categorically, because when more than one good potential match is available for each treated subject, there will be $k \geq 2$ such that some $1: k$ match leads to sharper estimates than do $1: 1$ matches, with little penalty in terms of bias. In the context of our coaching study, how much precision does each increment to the number of controls buy, and at what cost in terms of bias?

To appreciate the impact of the number of controls on the effect estimate's variability, consider that estimate in the context of a simple linear model. Attach numbers $1, \ldots, n$ to sample units; let $\mathbf{T}$ and $\mathbf{C}$ be the indices of the treated and the control group, respectively, so that $\mathbf{T} \cup \mathbf{C}=\{1, \ldots, n\}$ and $\mathbf{T} \cap \mathbf{C}=\emptyset$, and let $\mathbf{S}$ indicate a partition of the sample into matched and unmatched sets by mapping indices $\{1, \ldots, n\}$ of sample units to 0 , for unmatched units, or to positive integers $\{1, \ldots, S\}$ indicating matched sets. (The $s$ th matched set, $1 \leq s \leq S$, is then represented as $\mathbf{S}^{-1}[s]$.) Then the model represents responses of matched units [ $i$ for which $\mathbf{S}(i)>0$ ] as follows:

$$
\begin{aligned}
& Y_{i}= \begin{cases}\tau_{\mathbf{S}(i)}+\Delta_{\mathbf{S}(i)}+\varepsilon_{i}, & i \in \mathbf{T} \\
\Delta_{\mathbf{S}(i)}+\varepsilon_{i}, & i \in \mathbf{C},\end{cases} \\
& \quad \mathbf{E}(\varepsilon)=0, \operatorname{Cov}(\varepsilon)=\sigma^{2} \mathbf{I}, \sigma^{2}<\infty,
\end{aligned}
$$

where $\Delta_{1}, \ldots, \Delta_{S}$ are matched-set effects and $\tau_{1}, \ldots, \tau_{S}$ are treatment-control contrasts, one for each matched set.

Under model (1), in the sth matched set the average difference of treatment- and control-subgroup responses, $\bar{y}_{s t}-\bar{y}_{s c}$, unbiasedly estimates $\tau_{s}$, and weighted averages $\sum w_{s} \tau_{s}(w \geq 0$, $\left.\sum w_{s}=1\right)$ may be estimated with weighted averages of these matched-set response differences, $\sum w_{s} \hat{\tau}_{s}$. Given candidate stratifications $\mathbf{S}:\{0, \ldots, n\} \rightarrow\{0, \ldots, S\}$ and $\tilde{\mathbf{S}}:\{0, \ldots, n\} \rightarrow$ $\{0, \ldots, \tilde{S}\}$ with weightings $w$ and $\tilde{w}$, therefore, the quotient

$$
\begin{aligned}
R(\mathbf{S}, \tilde{\mathbf{S}}) \equiv & \left(\sum_{s=1}^{S} w_{s}^{2} \frac{\#\left(\mathbf{T} \cap \mathbf{S}^{-1}[s]\right) \cdot \#\left(\mathbf{C} \cap \mathbf{S}^{-1}[s]\right)}{}\right)^{1 / 2} \\
& \times\left(\sum_{\tilde{s}=1}^{\tilde{S}} \tilde{w}_{\tilde{s}}^{2} \frac{\#\left(\mathbf{T} \cap \tilde{\mathbf{S}}^{-1}[\tilde{s}]\right) \cdot \#\left(\mathbf{C} \cap \tilde{\mathbf{S}}^{-1}[\tilde{s}]\right)}{\left.\tilde{S}^{-1}[\tilde{s}]\right)}\right)^{-1 / 2}
\end{aligned}
$$

$[\#(A)=$ size or cardinality of $A]$ assesses the relative precision of estimates based on $\mathbf{S}$ and on $\tilde{\mathbf{S}}$. When $\mathbf{S}$ and $\tilde{\mathbf{S}}$ give rise to models of form (1) sharing a common value of $\sigma, R(\mathbf{S}, \tilde{\mathbf{S}})$ is the ratio of SD's of estimates of $w$ - and $\tilde{w}$-weighted averages of stratum treatment/control contrasts.

Comparisons based on this quotient favor $1: k$ matchings with larger values of $k$. Weighting strata in proportion to the number of treated subjects they contain is sometimes called effect of treatment on the treated (ETT) weighting; using ETT weights, $w_{s} \equiv \#(\{i \in \mathbf{T}: \mathbf{S}(i)=s\}) / \#(\{i \in \mathbf{T}: \mathbf{S}(i)>0\})$, and comparing each matching to a 1:1 matching, the relative precision quotients for $1: 1,1: 3,1: 5$, and $1: 7$ matchings are $1.00, .82, .77$, and .76. Matchings with multiple controls appear appreciably more precise.

The relative precision number $R(\mathbf{S}, \tilde{\mathbf{S}})$ does not depend on which subjects $\mathbf{S}$, or $\tilde{\mathbf{S}}$, groups together; for example, any two $1: 5$ matches $\mathbf{S}_{5}$ and $\mathbf{S}_{5}^{\prime}$ have $R\left(\mathbf{S}, \mathbf{S}^{\prime}\right)=1$. In contrast, biases attending to a stratification $\mathbf{S}$ are determined by $\mathbf{S}$ 's success at grouping like with like. Figure 1 shows standardized biases for the unmatched College Board coaching data and for optimal $1: 1,1: 3,1: 5$, and $1: 7$ propensity score matchings created with it.

Observe that the boxplots at the far left and far right of the figure are identical. This is no accident: The matched sets described by the rightmost boxplot exclude no controls and, in the computation of standardized biases, give all controls the same weight; the same occurs when covariate biases are calculated for the unmatched sample. In general, if controls are $K$ times as numerous as treated subjects, then adjustment using a $1: K$ matching amounts to no adjustment at all.

The pattern in Figure 1 appears in a number of contexts. It has led authors such as Dehejia and Wahba (1999) and Smith (1997) to conclude that whatever its advantages for variance, attempting to use most or all of the control reservoir invites sharp penalties in terms of bias. Full matching will turn out to involve a very different variance-bias tradeoff, however, making attractive another explanation of the penalties for increased use of controls seen in Figure 1: They reflect limitations inherent to fixed-ratio matching. 


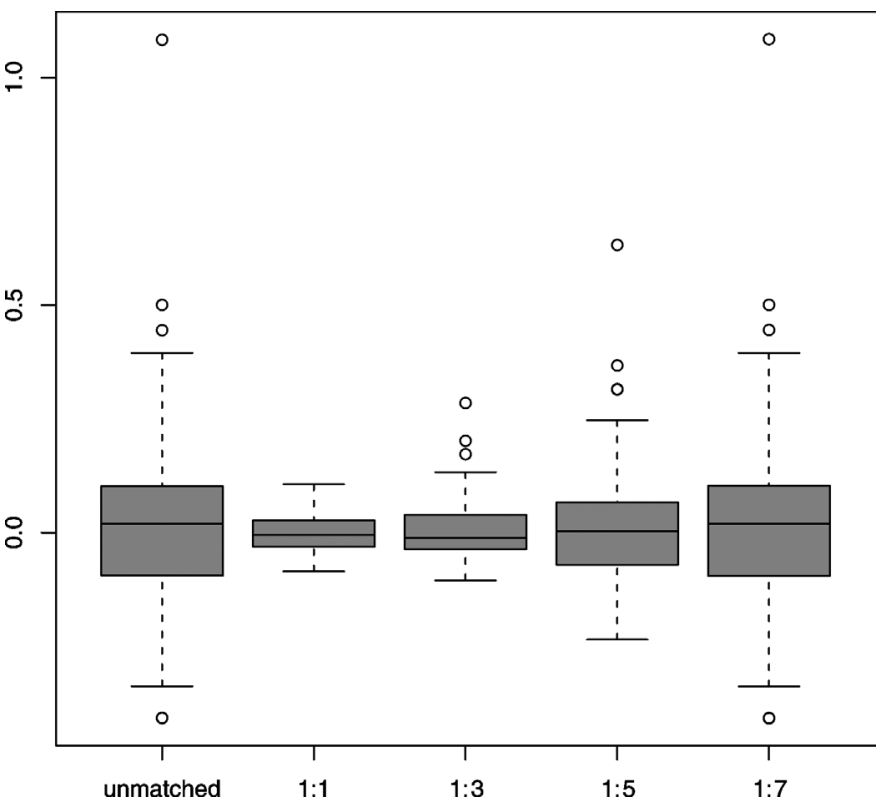

Figure 1. Covariate Imbalances in 1: $k$ Matching. Each boxplot represents standardized biases in the 99 categories of the 27 categorical covariates along with standardized bias in the propensity score (which in each plot is the uppermost outlier). Strictly speaking, the matching represented at far right is not a 1:7 matching but a blend of six 1:6 and 494 1: 7 matched sets.

\subsection{Full Matching: An Illustration}

Full matching subdivides a sample into a collection of matched sets consisting either of a treated subject and any positive number of controls or a control subject and any positive number of treated persons. These matchings stand in contrast to the $1: k$ matchings considered in the previous section. For example, one can readily verify that the optimum placement of the four women and five men in Table 3 into matched sets of one woman and one or two men matches A to $\mathrm{V}$ and $\mathrm{W}$, B to X, $\mathrm{C}$ to $\mathrm{Y}$, and $\mathrm{D}$ to $\mathrm{Z}$, with total cost 3.8. The optimal full match, depicted in Table 4, reduces this sum to 3.6. Coincidentally, it avoids matching any woman to a man whose grant funding differs from hers by more than a factor of 10 - a requirement that, with the help of full matching, could be insisted upon in the actual study on which the example is based. In the example problem, neither pair matching nor matching with one or two controls could have met such a requirement.

Rosenbaum (1991) introduced full matching, $\mathrm{Gu}$ and Rosenbaum (1993) did a simulation study of it, and Marcus (2000) made use of it to assess the Head Start compensatory education program.

Table 4. Full-Matching Solution to the Matching Problem Posed by Table 3

\begin{tabular}{|c|c|c|c|c|c|}
\hline \multicolumn{3}{|c|}{ Women } & \multicolumn{3}{|c|}{ Men } \\
\hline Subject & $\log _{10}($ grant $)$ & $\begin{array}{l}\text { Matched } \\
\text { set }\end{array}$ & Subject & $\log _{10}($ grant) & $\begin{array}{c}\text { Matched } \\
\text { set }\end{array}$ \\
\hline $\bar{A}$ & 5.7 & 1 & V & 5.5 & 1 \\
\hline B & 4.0 & 2 & W & 5.3 & 1 \\
\hline C & 3.4 & 2 & $X$ & 4.9 & 1 \\
\hline \multirow[t]{2}{*}{ D } & 3.1 & 2 & $Y$ & 4.9 & 1 \\
\hline & & & Z & 3.9 & 2 \\
\hline
\end{tabular}

\subsection{Matching to Use Every Control}

To judge from Figure 1, no way of matching coached to uncoached students at once balances all measured covariates and places each available control in some matched set. However, each matching in Figure 1 joins treated and control subjects in only a single, fixed ratio; full matching may introduce new possibilities. This section studies the optimal, in the sense of minimizing propensity score distances, full matching of the College Board sample. By its construction, such a matching cannot fail to use every available control, but its success or failure at imposing balance upon measured covariates remains to be seen.

For each pair $i \in \mathbf{T}$ and $j \in \mathbf{C}$, let a discrepancy $\delta_{i j} \in[0, \infty]$ be given: Small values of $\delta$ indicate desirable matches; large finite $\delta$ 's, matches to be avoided; infinite $\delta$ 's, matches that are forbidden. A full matching is a partition of all or part of the sample into one-one, one-many, and many-one matched sets, none of which includes forbidden pairings. Formally, by "full matching" let us understand a mapping $\mathbf{S}$ of $\mathbf{T} \cup \mathbf{C}$ into $\{0, \ldots, S\}$, $S$ a positive integer, such that each matched set $M=\mathbf{S}^{-1}[s]$ $(1 \leq s \leq S)$ satisfies $\min (\#(M \cap \mathbf{T}), \#(M \cap \mathbf{C}))=1$, and for all $i \in M \cap \mathbf{T}$ and $j \in M \cap \mathbf{C}, \delta_{i j}<\infty$. The size of a full matching $\mathbf{S}$ is the ordered pair $\left(\#\left(\mathbf{S}^{-1}[\{1, \ldots, S\}] \cap \mathbf{T}\right), \#(\mathbf{S})^{-1}[\{1, \ldots\right.$, $S\}] \cap \mathbf{C}$ ), indicating the number of treated and the number of control units that $\mathbf{S}$ places into matched sets. These definitions are substantially equivalent to those of Rosenbaum (1991).

Given a full-matching problem $\left(\mathbf{C}, \mathbf{T},\left\{\delta_{i j}\right\}\right)$, a full match $\tilde{\mathbf{S}}$ of size $(\mathbf{c}, \mathbf{t})$ that solves it is optimal among size $(\mathbf{c}, \mathbf{t})$ full matches if it minimizes net discrepancy,

$$
\sum_{i \in \mathbf{T}, \mathbf{S}(i)>0} \sum_{j \in \mathbf{C}, \mathbf{S}(i)=\mathbf{S}(j)} \delta_{i j},
$$

among all size $(\mathbf{c}, \mathbf{t})$ full matches $\mathbf{S}$ for $\left(\mathbf{C}, \mathbf{T},\left\{\delta_{i j}\right\}\right)$. An optimal full match is a minimizer of net discrepancy among size (\#(C),\#(T)) full matches, that is, full matches that discard no units.

In the present analysis, discrepancies $\delta_{i j}$ are based on the propensity score: For $i \in \mathbf{T}, j \in \mathbf{C}$,

$\delta_{i j} \equiv\left\{\begin{array}{l}\infty, \quad i, j \text { belong to different Race } \times \text { SES subclasses } \\ \left|\operatorname{logit}\left(\hat{e}\left(\mathbf{X}_{i}\right)\right)-\operatorname{logit}\left(\hat{e}\left(\mathbf{X}_{j}\right)\right)\right| \\ \quad \text { otherwise }\end{array}\right.$

where $\mathbf{X}$ is the vector of covariates and $\hat{e}\left(\mathbf{X}_{i}\right), \hat{e}\left(\mathbf{X}_{j}\right)$ are fitted propensity scores. The infinite distances force exact matching on race and father's education. An algorithm to find optimal full matches is described in the Appendix.

Full matching was very successful in removing bias due to observed covariates. The average within-stratum discrepancy between treateds and controls, understood as distance along the fitted score, is .05, and the optimal full match removes $99 \%$ of the bias in the fitted score. By contrast, average propensity distances in the optimal $1: 1,1: 3$, and $1: 5$ fixed-ratio matchings were $.04, .31$, and .69 , respectively, with propensity score bias reductions of $97 \%, 74 \%$, and $42 \%$. When the sample is partitioned according to the optimal full match, no covariate exhibits even a hint of association with treatment status; the CochranMantel-Haenszel $\chi^{2}$ statistics (see Sec. 1.2) are both nonsignificant and uniformly close to 0 . Evidently, full matching permits use of the control reservoir in its entirety, with no discernible penalty in terms of bias. 


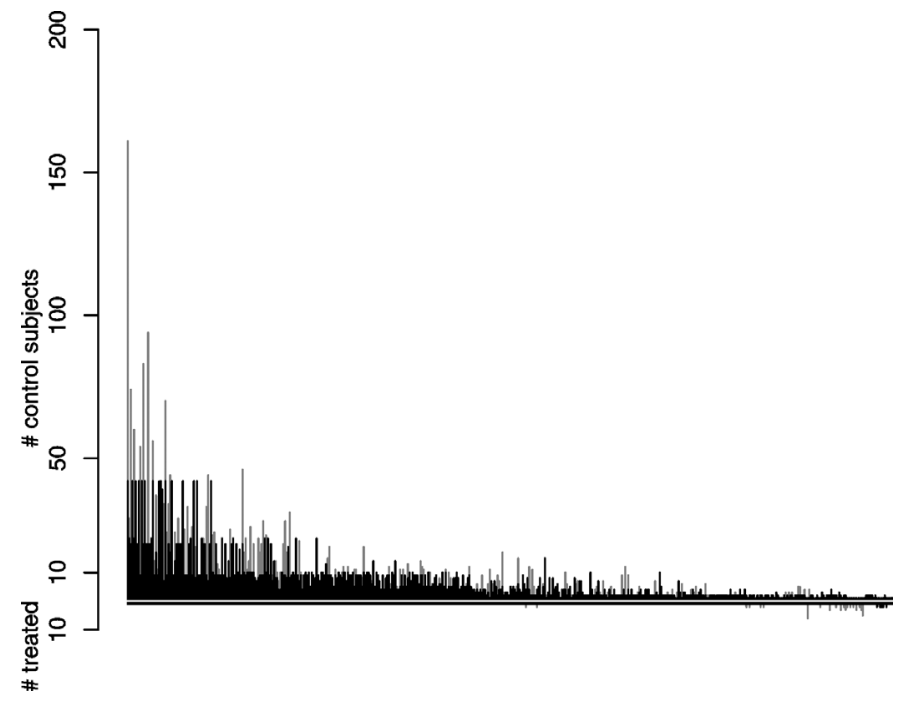

Figure 2. Superimposed Barplots Representing Stratum Sizes of the Optimal Full Match (gray bars, in background; 468 strata) and the Optimal $[.5,2]$ Full Match (in foreground; 491 strata). The contrast between the two illustrates that in full matching restrictions on treated-to-control ratios greatly reduce the number of matched sets that are unusually heavy with control or treated subjects. Vertically aligned bars represent a single matched set, with bar heights above the $x$ axis giving the number of controls in it and bar depth below the $x$ axis showing its share of treated subjects.

Variance is another issue. Relative to the optimal $1: 1$ match $\left(\mathbf{S}_{1}\right)$, the relative precision of the optimal full match is a disappointing .93. This may be better than the optimal pair match, but it is worse than every one-many match considered in Section 2.2. For instance, the optimal 1:3 matching $\mathbf{S}_{3}$-which substantially reduced covariate imbalances-had $R\left(\mathbf{S}_{1}, \mathbf{S}_{3}\right)=.82$. A use of full matching that contains variance as well as bias is described in the next section.

The optimal full match looks strikingly different from any fixed-ratio matching. Most notably, it contains some outlandishly large matched sets: as many as six treated subjects to a control; as many as 161 controls to a treated. Figure 2 represents the composition of its matched sets with stacked barplots. The black bars in the foreground of Figure 2 represent a matching that will be introduced in Section 3.2, but the gray bars in the background describe the optimal full matching. Adjoining upper and lower bars give the numbers of uncoached and of coached students in a single matched set, with matched sets arranged from left to right in order of increasing propensity score. This arrangement illustrates the natural tendency for subjects with high scores to be placed in many-one matched sets, while low propensity score subjects wind up in one-many substrata that are heavy with controls.

\section{FULL MATCHING WITH RESTRICTIONS}

The optimal full match uses all controls and balances every covariate, but some of its matched sets are too heavy with controls, and in others controls are quite sparse. The disparities stand behind the optimal full match's disappointing relative precision. And by altering so drastically the weighting of subjects implicit in the Race $\times$ SES subclassification, it engenders estimates of coaching effects that depend quite strongly on a particular propensity score specification. This section produces a full matching that is similar to the matching of Section 2.4 in that it balances available covariates without rejecting any controls. In terms of maintaining balance in the relative numbers of treated and control subjects in any matched set, on the other hand, it does a good deal better than that matching; this improvement increases precision, in the sense that it reduces standard errors of estimates of treatment effects. Some technical preliminaries are necessary, and it is best to begin with an illustration.

\subsection{Full Matching With Restricted Treated-to-Control Ratios}

Let us return for the moment to the matching problem of Table 3. As compared to the optimal pair matching, or to the optimal matching with one or two controls, the optimal full match given in Table 4 supports assessments of gender equity that have smaller bias, because it matches men and women more closely in terms of the one covariate being adjusted for. In terms of variance, however, it is actually worse than those matchings. Comparing to pair matching and using ETT weights for each comparison, the precision of a matching into three pairs and a $1: 2$ set is .97 , while a $1: 4,3: 1$ full matching (as in Table 4) has precision 1.27. One remedy for this is to constrain the result of one's full matching so that the ratios of the numbers of treated and of control subjects in each matched set are either homogeneous, as in the optimal pair matching, or at least relatively homogeneous, as they were in the one-to-two controls solution to the matching problem of Table 3 .

Suppose, for concreteness, that we seek a full matching such that in each matched set, the number of treated subjects divided by the number of controls ranges from about half up to about twice what that ratio is in the sample as a whole. For the gender equity matching problem, the overall ratio of treated (women) to control subjects (men) is $4: 5$, so we would seek individual matched sets with treated-to-control ratios of about $1: 2.5$ up to $1.6: 1$. Matched sets with 2.5 controls or 1.6 treated subjects are of course impossible, so we require a rounding convention. Let us be permissive rather than strict, interpreting the present requirement so as to permit matched sets with treated-to-control ratios of $1: 3$ up to $2: 1$. (By establishing the conventions in this way, we reduce the potential for inadvertently imposing a restriction that makes matching infeasible, as would occur in the equity matching problem if a restricting factor of .75 , rather than $1 / 2$, were placed on the reduction in the ratio of treated subjects to controls, and if the resulting upper limit of $1.67 \mathrm{con}-$ trols per treated subject were to be interpreted strictly.)

The full matching that minimizes costs while adhering, under this interpretation, to the half-to-twice restriction on the ratio of women to men is as follows: woman $\mathrm{A}$ is matched to men $\mathrm{V}$ and $\mathrm{W}$, and $\mathrm{B}$ to $\mathrm{X}$ and $\mathrm{Y}$; while women $\mathrm{C}$ and $\mathrm{D}$ are both matched to $\mathrm{Z}$. The restrictions lead to a somewhat greater total cost, 3.7 versus 3.6. Even with restrictions, however, full matching again makes it possible to avoid matching men and women whose $\log _{10}$ (Grant Funding) differs by more than 1 . At a small price, then, one secures a substantial improvement in precision: Writing $\mathbf{S}_{r}$ for the optimal full matching with constraints and $\mathbf{S}_{f}$ for Table 4's unconstrained optimal full matching, one has $R\left(\mathbf{S}_{r}, \mathbf{S}_{f}\right)=.82$.

Let us place these ideas into a suitable formalism. A matching $\mathbf{S}$ subdivides $\mathbf{U}$ if for all subject indices $i$ and $j, \mathbf{S}(i)=$ 
$\mathbf{S}(j)$ entails $\mathbf{U}(i)=\mathbf{U}(j)$. When $\mathbf{S}$ subdivides $\mathbf{U}$, for each matched set $M$ of $\mathbf{S}$ there is a stratum $U$ of $\mathbf{U}$, that is, $U=$ $\mathbf{U}^{-1}[s]$ for some $s \geq 1$, such that $M \subseteq U$. Given a stratification $\mathbf{U}$, call the ratio of treated subjects to controls in $U$ the $\mathbf{U}$-treatment odds for stratum $U$. When $\mathbf{S}$ subdivides $\mathbf{U}$, a matched set $M$ of $\mathbf{S}$ has both $\mathbf{S}$-treatment odds, $d \mathbf{S}_{(M) \text {, }}$ and $\mathbf{U}$-treatment odds, $d^{\mathbf{U}}(M)$, namely the $\mathbf{U}$-treatment odds for the stratum $U$ of $\mathbf{U}$ that contains it. In the gender equity example, the null stratification $\mathbf{U}_{0}:\{\mathrm{A}, \mathrm{B}, \mathrm{C}, \mathrm{D}, \mathrm{V}, \mathrm{W}, \mathrm{X}$, $Y, Z\} \mapsto\{1\}$ is subdivided by $\mathbf{S}_{r}$. Regarding women as treated and men as control subjects, the $\mathbf{U}_{0}$-treatment odds for $\mathbf{U}_{0}$ 's lone stratum, $d^{\mathbf{U}_{0}}(\{\mathrm{~A}, \mathrm{~B}, \mathrm{C}, \mathrm{D}, \mathrm{V}, \mathrm{W}, \mathrm{X}, \mathrm{Y}, \mathrm{Z}\})$, are $4: 5$, as are the $\mathbf{U}_{0}$-treatment odds in each of $\mathbf{S}_{r}$ 's matched sets; but $\mathbf{S}_{r}$ 's three matched sets have $\mathbf{S}_{r}$-treatment odds of $d^{\mathbf{S}_{r}}(\{\mathrm{~A}, \mathrm{~V}, \mathrm{~W}\})=$ $1: 2, d^{\mathbf{S}_{r}}(\{\mathrm{~B}, \mathrm{X}, \mathrm{Y}\})=1: 2$, and $d^{\mathbf{S}_{r}}(\{\mathrm{C}, \mathrm{D}, \mathrm{Z}\})=2: 1$.

A matching $\mathbf{S}$ that subdivides $\mathbf{U}$ respects a thickening cap of $u, u \geq 1$, if the $\mathbf{S}$ - and $\mathbf{U}$-treatment odds obey the relation

$$
d^{\mathbf{S}}(M) \leq \begin{cases}\left\lceil u d^{\mathbf{U}}(M)\right\rceil: 1, & u d^{\mathbf{U}}(M)>1 \\ 1:\left\lfloor\left(u d^{\mathbf{U}}(M)\right)^{-1}\right\rfloor, & u d^{\mathbf{U}}(M) \leq 1\end{cases}
$$

for each matched set $M$ of $\mathbf{S}$. Such an $\mathbf{S}$ nowhere increases the ratio of treated to control subjects to more than roughly $u \cdot 100 \%$ of what it would have been under $\mathbf{U}$. As a subdivision of the null stratification $\mathbf{U}_{0}$, the restricted full matching $\mathbf{S}_{r}$ respects a thickening cap of 2 .

Similarly, the subdivision of $\mathbf{U}$ into $\mathbf{S}$ conforms to a thinning cap of $l$ if $0 \leq l \leq 1$ and for each matched set $M$ of $\mathbf{S}$,

$$
d^{\mathbf{S}}(M) \geq \begin{cases}\left\lfloor l d^{\mathbf{U}}(M)\right\rfloor: 1, & l d \mathbf{U}_{(M)}>1 \\ 1:\left\lceil\left( l d \mathbf{U}_{\left.(M))^{-1}\right\rceil,}\right.\right. & l d d^{\mathbf{U}}(M) \leq 1 .\end{cases}
$$

As a subdivision of $\mathbf{U}_{0}, \mathbf{S}_{r}$ holds to a thinning cap of $1 / 2$.

An $[l, u]$-subdivision of $\mathbf{U}$ is a subdivision of $\mathbf{U}$ respecting a thinning cap of $l$ and a thickening cap of $u$. An optimal $[l, u]$ subdivision of $\mathbf{U}$ is an $[l, u]$-subdivision of $\mathbf{U}$ with minimal net discrepancy [cf. (3)] among full matches that subdivide $\mathbf{U}$ and conform to thinning and thickening caps of $l$ and $u$. $\mathbf{S}_{r}$ is an optimal [.5, 2]-subdivision of $\mathbf{U}_{0}$.

\subsection{Restricted Full Matching for the Board Sample}

Now let $\mathbf{U}$ denote the Race $\times$ SES subclassification (Sec. 1.2). We seek an optimal $[l, u]$-subdivision of $\mathbf{U}, l<1$ and $u>1$, that adequately balances each covariate while keeping $l$ and $u$ as close to one as is consistent with this aim.

One-half and two are a natural pair of caps with which to start: Alter the treatment odds within strata, they say, by no more than a factor of 2. Against the optimal $[.5,2]$ full match, testing each of the 27 covariates separately using statistics of the Mantel-Haenszel (MH) type (cf. Sec. 1.2) yields no results of significance at the nominal .05 level; only with the parents' income variable is there a hint of association $\left(M^{2} / \mathrm{df}=8.9 / 4\right.$, $p=.06$ ). Alternatively, the battery of tests may be directed at subjects without missing covariate data. The 27 additional MH tests that exclude those matched sets containing a subject missing data on the relevant covariate also fail, for the most part, to reject null hypotheses of no association. The exceptions are a test giving some thin evidence of association between the parents' income variable and treatment status, with

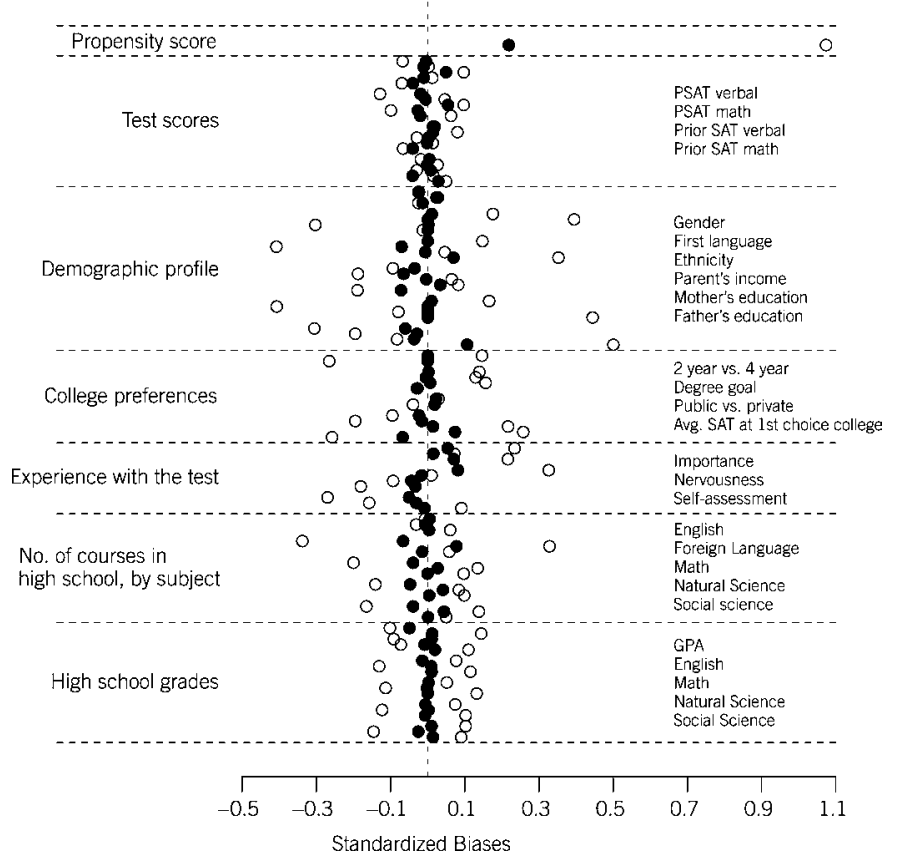

Figure 3. Standardized Biases Without Stratification or Matching, Open Circles, and Under the Optimal [.5, 2] Full Match, Shaded Circles.

$M^{2} / \mathrm{df}=7.0 / 3$ and $p=.07$, and a significant test of association between treatment status and years of foreign language, with $M^{2} / \mathrm{df}=4.8 / 1$ and $p=.03$. In short, of 27 covariates, one associates with treatment status at the .1 level, but not at the .05 level, and another may appear associated with treatment status at the .05 , but not at the .01 , level, depending on how one handles missing values. One might expect similar results under random assignment. Figure 3 depicts the optimal $[.5,2]$ full match's treatment-control group balance in each category of each of the 27 covariates, also showing imbalances prior to matching or stratification, for comparison.

In this application, a search among full matches optimal relative to various thinning and thickening caps terminated with the optimal $[.5,2]$ full match. The search varied the thickening cap $u$ first, before imposing a thinning cap, because under ETT weightings of stratum effects, $u$ 's impact on precision is greater than that of the thinning cap $l$ : It is readily confirmed using (2) that replacing a $1: 1$ and a $1: 5$ stratum with two $1: 3$ strata yields much more precision than does replacing a $1: 10$ and a $1: 50$ stratum with two $1: 30$ strata. When $\mathbf{U}$ is optimally subdivided with thickening caps decreasing from $\infty$ to $10(=10 / 1)$, to $5(=10 / 2)$, to $10 / 3$, to $10 / 4$ and then to $10 / 5$ or 2 , ETTweighted precision increases while none of the $54 \mathrm{MH}$ statistics for the resulting full matches become significant at the .1 level. The optimal $[0,10 / 6]$ full matching is still more precise, but because it has MH statistics that are significant at the .1 and .05 levels, we fix the thickening cap at 2 .

This leads us to compare optimal $[.2,2],[.3,2], \ldots$, and $[.7,2]$ full matchings. The first three of these have no MH statistics that are significant at the .1 level, and the last two each have at least two MH statistics significant at the .05 level. Recall that the optimal $[.5,2]$ matching had one MH statistic significant at the .05 level and two more significant at the .1 level, an acceptably small degree of confounding of covariates with 
0
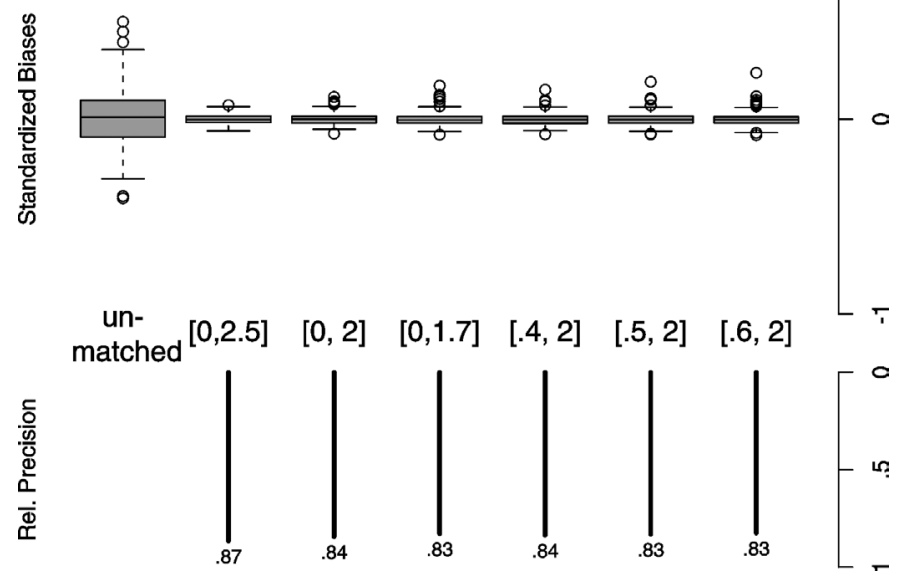

Figure 4. Standardized Biases and Relative Precision [R(., unconstrained full match)] of Optimal Stratifications With Variously Constrained Match Ratios.

treatment status. These comparisons lead us to prefer a thinning cap of .5. (Had we selected first a thinning and then a thickening cap rather than the reverse, this procedure would have led us instead to the optimal $[.6,2.5]$ full match.) Figure 4 displays standardized biases and relative precisions $R(\cdot, \mathbf{U})$, where $\mathbf{U}$ is the optimal $1: 1$ match, for optimal $[l, u]$ full matchings with various $l$ and $u$.

\subsection{Reduced Sensitivity to Model Specification}

The model here used to estimate propensity scores lacks interaction terms among its independent variables and involves no auxiliary modeling of data missingness. This puts it among the simplest of models one might use for propensity score estimation; it was chosen for this reason. Certainly, more elaborate propensity score models have been used; Rosenbaum and Rubin (1984), for example, employed a stepwise variable selection procedure to select main effects and then interaction terms, and D'Agostino and Rubin (2000) modeled item missingness explicitly, fitting their model using the EM algorithm.

The matching strategy taken here, stratifying on variables strongly predictive of treatment status before full matching with restrictions, aimed to limit the dependence of the analysis on any one specification of the treatment assignment model. To assess its success at this, a more saturated propensity score model was fit. As right-hand-side variables, this model has eight interactions and 17 main effects of the original variables, chosen by backward-forward stepwise variable selection. Using this model's fitted propensity score, evaluations of thickening and then thinning caps lead one to prefer an optimal [.4,2] full match. Call this new matching $\mathbf{S}_{2}$, and the matching selected in Section 3.2, $\mathbf{S}_{1}$.

Both full matches use all of 3,494 controls, and in most cases the two matchings place these control subjects into matched sets of very similar sizes: $20 \%$ of the 3,494 go into matched sets of precisely the same size; for $72 \%$ of controls $j$, the $\mathbf{S}_{2}$-treatment odds of $j$ 's $\mathbf{S}_{2}$ stratum are no more than $4 / 3$, and no less than $3 / 4$, of the $\mathbf{S}_{1}$-treatment odds of $j$ 's $\mathbf{S}_{1}$ stratum. Because a subject's contribution to our effect estimates is determined by the configuration of the matched set into which it is placed, it should be no surprise that the two full matchings lead to similar estimates of the coaching effect.

\section{ESTIMATING TREATMENT EFFECTS}

To estimate treatment effects, a model such as (1) must be supplemented with a causal formalism and appropriate causal assumptions. For this analysis, the most natural setup is that of Rubin (1977), who posits random variables $Y_{t}$ and $Y_{c}$ both for outcomes under the control condition and for outcomes under the treatment condition. Adding the assumption that these variables are conditionally independent of the treatment assignment variable $(Z)$ given the covariates $(\mathbf{X})$ makes inference about treatment effects possible.

Using ETT weighting to combine by-stratum treatment control differences, the $[.5,2]$ matching leads to aggregate contrasts of 26 points on the math section and 1 point on the verbal. Under causal assumptions as presently discussed, these estimate effects of coaching on the coached. Using model (1), the accompanying standard errors are 5 and 5 points. By contrast, the unadjusted differences of treated and control group means were $41( \pm 5)$ and $9( \pm 5)$ points.

As one might expect, those matchings that fail to reduce discernible biases to an indiscernible level give higher effect estimates. For example, the nearly fixed-ratio matching that respects Section 1.2's subclassification while using all controls, that is, the optimal $[1,1]$ subdivision of the Race $\times$ SES subclassification, offers estimates of $30( \pm 5)$ and $2( \pm 5)$. Of all matchings that respect the Race $\times$ SES subclassification, this had the most favorable relative precision quotients; yet its estimated standard errors are only negligibly smaller than those of the $[.5,2]$ match, while its poorer balance translates to apparent biases of one or more standard errors in estimates of the coaching effect. Conversely, those matchings that did reduce observed biases to indiscernibility gave lower estimates. The optimal [.6, 2.5] full match of Section 3.2 gives estimates of $23( \pm 5)$ and $0( \pm 5)$, and Section 3.3's [.4, 2] full match leads to estimates of $23( \pm 5)$ and $0( \pm 5)$.

\subsection{Heterogeneity of Coaching Effects}

Unlike both pair matching and analysis of covariance, full matching's estimates and standard errors do not assume treatment effects to be the same across units; they average estimates of individual treatment effects that can, in principle, be quite different. This is especially advantageous in a coaching study based on a representative national sample, since coaching programs differ widely in duration, rigor, and approach. As a relaxation of the constant-effect model, consider the hypotheses of 12 math and 12 verbal effects, one for each Race $\times$ SES subclass. By dint of the exact matching on race and father's education level, the matched-set coaching effects are nested within subclass coaching effects, and the three models-the constanteffect model, the 12-effects model, and model (1), with its 494 separate treatment effects-can be compared by an analysis of variance (ANOVA). In either the math or the verbal case, $F$ tests based on the ANOVA reject the constant-effect model in favor of either the 12-effects model or model (1), and the 12-effects model cannot be rejected from within (1). The hypothesis that there is a single, constant treatment effect is untenable. Granted, given the variety among interventions here 
grouped together as "treatments," there is little to recommend such hypothesis; yet previous coaching studies have often been analyzed using regression models that are built upon it.

\subsection{Very Large and Very Small Coaching Effects: Which Are More Plausible?}

Is it strange that structured, extended training for the SAT should produce, on the average, no more than a negligible benefit in verbal scores? In interpreting this result, one should bear in mind that the control condition is not the absence of preparation for the SAT. In this observational study, "not coached" means only "did not participate in a program of preparation for the SAT-I that was held outside of school." Controls may have, and by and large did, practice and otherwise prepare for the tests on their own or in school (Powers and Rock 1999); it is possible that these preparations were sometimes more effective than formal coaching. Indeed, our matching also facilitates estimating what effect the treatment would have had on the controls, were they treated, and the results of this calculation suggest that some controls benefited by selecting alternative test preparations: The effect of treatment for the controls is estimated at $3 \pm 7$ points on the math section and $-8 \pm 7$ points on the verbal. Evidently, whether and to what degree coaching is beneficial varies greatly from student to student.

Our analysis's allowance for heterogeneous coaching effects permits it to speak more directly to the coaching companies' claims than did Powers and Rock's analyses. Recall that the Princeton Review claims its students' average benefit is 140 points in the combined score (Princeton Review 2004), and that Kaplan Educational Centers have said that students benefit from their courses, on average, by 120 points (Zehr 2001). Further, a Kaplan spokesman has argued that very different gains are to be expected from Kaplan's programs as opposed to those of many of its competitors; shorter, cheaper, and untested programs abound, and smaller benefits are to be expected from them (Kolata 2001). The multiple-regression type of analysis favored by Powers and Rock cannot speak directly to this argument, as they assume uniform treatment effects, but the matching-based analysis of this article can.

At least $41 \%$ of coached students in the College Board sample had been coached by either Kaplan Educational Centers or the Princeton Review. Consider the hypothesis that Kaplan and the Princeton Review offer varying coaching benefits that average to 120 points, say. To permit a robust test of this hypothesis, let us supplement it with the unlikely assumption that all other companies' coaching benefits average to 0 . Even with models of form (1), which grant each matched set its own treatment effect, the upper $95 \%$ confidence bounds for the math and verbal effects (of coaching upon the coached) are about 37 and 11 points. Combined, these fall short of the 49-point overall average effect that Kaplan's and the Princeton Review's claims would, at a minimum, entail. The hypothesis is rejected.

\section{DISCUSSION: UPDATING THE LIMITATIONS OF MATCHING}

Observational studies compare persons who received a specified treatment to others who did not, adjusting for pretreatment differences between treated and comparison groups. Broadly, these adjustments are effected either by regression modeling or by stratification. Regression adjustments assume that we know or can reliably discern patterns relating pretreatment, treatment, and response variables, and require the statistician to specify and fit a corresponding statistical model. Adjustment by stratification assumes only that treatment and control groups sufficiently alike in terms of pretreatment characteristics are comparable in terms of response to treatment; but it requires the statistician to make precise what it means for groups to be sufficiently alike prior to treatment, and it requires a method for grouping subjects into sufficiently uniform blocks.

Matching and stratification are old and trusted methods of adjustment for observational studies, but the difficulty of implementing them led earlier practitioners to prefer regression. Cochran (1972) warned that in "larger studies... matching becomes impractical." In smaller and more manageable settings, Cochran recommended stratification as adjustment only for the one or two most important variables. Adjusting by matching or stratification for more than a few variables seemed hopeless: In one widely cited case, Chapin (1947) started with 671 treated subjects and 523 controls but found only 23 treatedcontrol pairs matching exactly on six categorical covariates. Citing these concerns, Cochran concluded: "[I]f there are say $4 x$ variables... [then our] recourse is to model construction and analysis based on the model."

Cochran gave this pessimistic assessment some 30 years ago. Advances since then have made observational data no more amenable to exact matching than they ever were, but the introduction of propensity scores (Rosenbaum and Rubin 1984) has greatly facilitated approximate matching on many variables; results possible with propensity scores and optimal full matching stand in striking contrast with Cochran's. In analytic studies, Cochran (1965) studied stratification on a single variable for treatment and control groups no more than a standard deviation apart. The schemes he studied removed as little as $57 \%$ and no more than $89 \%$ of the bias along the covariate. In the present case study, optimal full matching removes as much as $99 \%$ of the bias along a propensity score on which the treated and control means are separated by 1.1 SD's. In so doing, we have seen, it reduced to insignificance biases along 27 covariates, and it made use of more, not less, of the data than did regressionbased analyses. With flexible matching routines increasingly available, will regression adjustment for observational studies soon be obsolete?

\section{APPENDIX: OLSEN'S ALGORITHM: A UNIFIED APPROACH TO OPTIMAL MATCHING}

This appendix presents the algorithm used in this article to create optimal matchings. We employ a variant of the approach of Rosenbaum (1991), who presented (sec. 7) a general method for translating full-matching problems into network flow problems, which can in turn be solved efficiently using any of several widely available algorithms. According to Section 2.4, full-matching problems may be associated with triples (T, $\left.\mathbf{C},\left\{\delta_{i j}\right\}\right)$. A full-matching problem is feasible if it has a solution with finite total discrepancy. An optimal solution is one in which the average discrepancy within matched sets, $\sum_{s} \sum_{i \in \mathbf{T}, j \in \mathbf{C}: \mathbf{S}(i)=\mathbf{S}(j)=s} \delta_{i j} /\left(\sum_{s} \#\{(i, j) \in \mathbf{T} \times \mathbf{C}: \mathbf{S}(i)=\mathbf{S}(j)=s\}\right)$, is as small as it would be under any other solution. Rosenbaum (1991) represented such a problem as a graph with a node for every treated and every control, plus a node called "Source" and another called "Drain," 
with edges from Source to each treated node, from $i$ to $j$ when $\delta_{i j}<\infty$, and from every control node $j$ to Drain. A network flow problem is associated with this graph in such a way that optimal flows through the network correspond to optimal full matchings.

By constrained full-matching problem, let us understand a fullmatching problem $\left(\mathbf{T} ; \mathbf{C} ;\left\{\delta_{i j}\right\}\right)$ accompanied by upper and lower limits $(L, U)$ on the number of controls per treated and upper and lower limits $(\tilde{L}, \tilde{U})$ on the number of treateds per control; a constrained problem $\left(\mathbf{T} ; \mathbf{C} ;\left\{\delta_{i j}\right\} ; L, U, \tilde{L}, \tilde{U}\right)$ is feasible if there exists a solution of the problem $\left(\mathbf{T} ; \mathbf{C} ;\left\{\delta_{i j}\right\}\right)$ which respects the constraints. The problem of finding an optimal $[l, u]$-subdivision of a stratification $\mathbf{U}$, if one exists, is easily translated to a sequence of constrained full-matching problems - one for each stratum of $\mathbf{U}$. In the applications discussed previously, controls outnumber treated subjects in each stratum, with the consequence that $\tilde{L} \leq 1$ for each constrained full matching problem that had to be solved.

To handle constrained full-matching problems with $\tilde{L}=1$ or 0 , Olsen (1997, chap. 6) modified Rosenbaum's (1991) algorithm in several important ways. First, in addition to Source and Drain nodes and a node for each study subject, there is an additional node, labeled "Overflow," with edges connecting it to each treated and to each control node; there is no cost for sending units of flow along these edges. Second, a negligible amount $\varepsilon>0$ is added to each discrepancy $\delta_{i j}$. In Olsen's network flow problem, the upper and lower capacity limits for edges of various types are as follows: for edges from treateds to controls, $[0,1]$; for an edge from Source to a treated, say $i,[0, U]$; for an edge from a treated, $i$, to Overflow, $[0, U-L]$; for an edge from a control, $j$, to Overflow, $[0, \tilde{U}-1]$; and for an edge from a control, $j$, to Drain, $[0,1]$. The supply (exogenous inflow) is 0 at each node except Source, Drain, and Overflow, at which supplies are $U \cdot \# \mathbf{T},-p \cdot \# \mathbf{C}$, and $p \cdot \# \mathbf{C}-U \cdot \# \mathbf{T}$, respectively. Here, $p$ is the proportion of available controls that are to be matched to treated subjects; thus $p \leq 1$, and $p<1$ only if $\tilde{L}=0$. The constrained full-matching problem is feasible if and only if the Olsen network flow associated with it is feasible. In this case, an optimal flow through the network corresponds to a full match that is optimal among appropriately constrained full matches.

To implement Olsen's algorithm, I created functions calling the RELAX-IV network optimization routine of Bertsekas and Tseng (1994) from the R environment (r-project.org); this code is bundled together in an add-on package to R, "optmatch," information about which can be found on my Web site.

\section{[Received April 2003. Revised January 2004.]}

\section{REFERENCES}

Agresti, A. (1990), Categorical Data Analysis, New York: Wiley.

Bertsekas, D. P., and Tseng, P. (1994), "RELAX-IV: A Faster Version of the RELAX Code for Solving Minimum Cost Flow Problems," Technical Report P-2276, Massachusetts Institute of Technology, available at mit.edu/ dimitrib/www/noc.htm.
Briggs, D. (2001), "The Effect of Admissions Test Preparation: Evidence From NELS:88," Chance, 14, 10-21.

Campbell, D., and Stanley, J. (1966), Experimental and Quasi-Experimental Designs for Research, Boston: Houghton Mifflin.

Chapin, F. (1947), Experimental Designs in Sociological Research, New York: Harper \& Row.

Cochran, W. G. (1965), "The Planning of Observational Studies of Human Populations," Journal of the Royal Statistical Society, 128, 234-266.

(1972), "Observational Studies," in Statistical Papers in Honor of George Snedecor, Iowa State University Press, pp. 77-90.

D'Agostino, R., Jr., and Rubin, D. (2000), "Estimating and Using Propensity Scores With Partially Missing Data," Journal of the American Statistical Association, 95, 749-759.

Dehejia, R., and Wahba, S. (1999), "Causal Effects in Nonexperimental Studies: Reevaluating the Evaluation of Training Programs," Journal of the American Statistical Association, 94, 1053-1062.

$\mathrm{Gu}$, X., and Rosenbaum, P. (1993), "Comparison of Multivariate Matching Methods: Structures, Distances, and Algorithms," Journal of Computational and Graphical Statistics, 2, 405-420.

Kolata, G. (2001), "Admissions Test Courses Help, but Not So Much, Study Finds," The New York Times, 1, 16.

Mantel, N. (1963), "Chi-Square Tests With One Degree of Freedom: Extensions of the Mantel-Haenszel Procedure," Journal of the American Statistical Association, 58, 690-700.

Marcus, S. M. (2000), "Estimating the Long-Term Effects of Head Start," in Into Adulthood: A Study of the Effects of Head Start, eds. S. Oden, L. J. Schweinhart, and D. P. Weikart, Ypsilanti, MI: High/Scope Press, Chap. F, pp. 179-200.

Ming, K., and Rosenbaum, P. (2000), "Substantial Gains in Bias Reduction From Matching With a Variable Number of Controls," Biometrics, 56, 118-124.

Olsen, S. (1997), "Multivariate Matching With Non-Normal Covariates in Observational Studies," Ph.D. thesis, University of Pennsylvania.

Powers, D., and Rock, D. (1999), "Effects of Coaching on SAT I: Reasoning Test Scores," Journal of Educational Measurement, 36, 93-118.

Princeton Review (2004), "SAT Classroom Courses for Class of 2005," available at princetonreview.com.

Rosenbaum, P. (1984), "The Consequences of Adjustment for a Concomitant Variable That Has Been Affected by the Treatment," Journal of the Royal Statistical Society, Ser. A, 147, 656-666.

(1991), "A Characterization of Optimal Designs for Observational Studies," Journal of the Royal Statistical Society, 53, 597-610.

Rosenbaum, P., and Rubin, D. (1984), "Reducing Bias in Observational Studies Using Subclassification on the Propensity Score," Journal of the American Statistical Association, 79, 516-524.

(1985), "Constructing a Control Group Using Multivariate Matched Sampling Methods That Incorporate the Propensity Score," The American Statistician, 39, 33-38.

Rubin, D. B. (1977), "Assignment to Treatment Group on the Basis of a Covariate (Corr: V3 P384)," Journal of Educational Statistics, 2, 1-26.

Smith, H. (1997), "Matching With Multiple Controls to Estimate Treatment Effects in Observational Studies," Sociological Methodology, 27, 325-353.

Zehr, M. (2001), "Study: Test-Preparation Courses Raise Scores Only Slightly," Education Week, available at ed-week.com. 